\title{
Opportunities and Challenges in Developing Technology-Based Social Skills Interventions for Adolescents with Autism Spectrum Disorder: A Qualitative Analysis of Parent Perspectives
}

\author{
Roxanne N. Rashedi ${ }^{1} \cdot$ Kemberlee Bonnet $^{2} \cdot$ Rebecca J. Schulte $^{2} \cdot$ David G. Schlundt $^{2} \cdot$ Amy R. Swanson $^{3}$. \\ Amy Kinsman $^{3} \cdot$ Nicole Bardett $^{3} \cdot$ Pablo Juárez $^{3} \cdot$ Zachary E. Warren $^{3} \cdot$ Gautam Biswas $^{1} \cdot$ Maithilee Kunda $^{1}$
}

Accepted: 28 September 2021 / Published online: 12 October 2021

(c) The Author(s), under exclusive licence to Springer Science+Business Media, LLC, part of Springer Nature 2021

\begin{abstract}
Interest continues to be high in technology-based interventions for individuals with autism spectrum disorder (ASD). Understanding the preferences and challenges of technology use among individuals with ASD can inform the design of such interventions. Through 18 interviews with parents, we used an iterative inductive-deductive approach to qualitative analysis and explored uses of technology for social skills development among adolescents with ASD. Our findings include parents' observations about their adolescent's preferences in types of technology devices and digital content, as well as both positive and negative effects of technology use on mood and behavior. Parents highlighted several avenues of technological preferences and risks that may inform intervention design, enhance user engagement, and capitalize on users' strengths while buttressing areas for growth.
\end{abstract}

Keywords Qualitative · Technology · Autism

\section{Introduction}

Many individuals with autism spectrum disorder (ASD) have difficulties with theory of mind (ToM) and social skills, which are essential for navigating daily aspects of learning, communication, and social interaction (Frith, 1994; Peterson et al., 2009). Theory of mind refers to a broad constellation of cognitive abilities that involve representing and reasoning about other people's mental states, such as their beliefs, emotions, intentions, and desires. Social skills, i.e., cognitive and communicative skills deployed in any kind of interpersonal interaction, both influence and are influenced by an

Maithilee Kunda

mkunda@vanderbilt.edu

1 Vanderbilt University, School of Engineering, 2301

Vanderbilt Pl, Nashville, TN 37235, USA

2 Department of Psychology and Human Development, Vanderbilt University, 230 Appleton Pl, Nashville, TN 37203, USA

3 Vanderbilt Kennedy Center, Vanderbilt University, 110 Magnolia Cir, Nashville, TN 37203, USA individual's theory of mind abilities (Hughes \& Leekam, 2004).

Although decades of ASD-focused research have explored educational interventions to improve individuals' theory of mind abilities and social skills, there is still a need for more varied and effective interventions for different target skills and populations, as well as for more robust practices surrounding their design (Fletcher-Watson et al., 2014; Rao et al, 2008; Reichow \& Volkmar, 2010). For instance, successful theory of mind interventions could have very high potential impact, given the prevalence of theory of mind difficulties observed in children and adults with ASD (Fletcher-Watson et al., 2014), and developmental research that identifies bidirectional links between theory of mind and everyday social functioning (Hughes \& Leekam, 2004). However, a rigorous review of theory of mind interventions for ASD published in 2014 found that, while there was evidence that various theory of mind abilities could be improved in in some fairly narrow contexts, more evidence is needed within each theory of mind subskill category as well as across different subsets of the population: "different age and ability levels require support developing different sub-skills and mapping out these relationships would be of value" (Fletcher-Watson et al., 2014, p. 22). 
As in many areas of education, interactive technologies-including TV/videos, games, classroom-based applications, social media, etc.-offer promise and challenge as a potential medium for interventions for users with ASD. It is this fundamental tension that motivates the current study. On the one hand, using technologies to deliver interventions can offer enormous benefits in terms of scalability and reach, user engagement, new modes of social interaction and socially motivated achievement (e.g., social media, online content creation), and personalized interactions delivered through "intelligent" AI-based systems. On the other hand, while users with ASD often enjoy and accept various technologies, including being able to access new modes of online social interaction, concerns loom that too much time on devices can negatively impact users' mood or social functioning, and that users' time on devices is time taken away from real-world communication and social interaction (Odom et al., 2015).

These concerns also overlay more general technologyrelated risks such as inequalities in access or the "digital divide" (Dolan, 2016), questions of data use ethics and user privacy (Pardo \& Siemens, 2014), issues of credibility, and information literacy with the avalanche of information now available online (Metzger et al., 2015). Furthermore, many of these concerns apply to all users of technology, children and adults, with or without atypical cognitive or developmental conditions.

However, we believe that concerns about the effects of technology use are uniquely salient at the specific intersection of (a) theory of mind and social skills interventions for individuals with ASD, and (b) the profound effects that technology now imposes on a person's social world. In others words, given that technology-based theory of mind and social skills interventions are going to play a significant role for individuals with ASD in the coming years and decades, what can we learn about the opportunities and challengesthe push and pull—of how such technologies may impact the lives of their users?

As technology designers, we approach this question from the perspective of a participatory design process, such that users with ASD and their close proxies (e.g., parents) are involved in developing the technology (Spiel et al., 2019). With the support of a federally funded grant in the United States, we are working to design, implement, and evaluate a new educational game for helping adolescents with ASD improve their ToM and social reasoning skills. Motivating our work are observations of gaps in the intervention literature, e.g., as made in a review of social skills interventions, albeit from several years ago, which observed that far fewer intervention studies til that time had focused on adolescents and adults, in comparison to studies looking at younger children (Reichow \& Volkmar, 2010, p. 162); and another observation in a review of ToM interventions pointing out that, "Perhaps surprisingly, a minority of included studies focused explicitly on training ToM skills" as opposed to ToM precursor skills like emotion recognition (FletcherWatson et al., 2014, p. 22).

Our target users are adolescents with ASD who have challenges in areas of social skills and communication and who need only limited support in areas of reading comprehension and/or other academic skills. Our interdisciplinary team includes expertise in clinical psychology, special education, cognitive science, STEM educational technologies and educational data mining, user experience research, and qualitative methods.

Developing an inclusive, well-implemented educational ToM and social skills game for adolescents with ASD calls for the meaningful involvement of stakeholders early on in the design process (Durlak, 2015). In particular, shifting away from a user-centered design process, we adopt a participatory design process-i.e., rather than designing for users, we aim to design with users by inviting them to contribute their own interests, needs, and perspectives as effectual inputs into the design process (Sanders, 2002; Spiel et al., 2019). Our design process draws from participatory experiences, experiences pointing to the attitudes about the people whom our educational game-based intervention aims to serve. In this sense, our project is grounded in the notion that all users, including close proxies to users (e.g., parents, teachers, friends, and other stakeholders and community members), have something important to contribute to the design process (Sanders, 2002).

The adoption of inclusive, participatory design processes adds value to the development of technologies for individuals with ASD (Zhu et al., 2019). Such value includes better pinpointing specific needs and desires of intended end users (Putnam et al., 2019); recognizing, enhancing, and benefiting from diverse and neurodiverse perspectives (FletcherWatson et al., 2018); and promoting increased agency of end-users in the design phase of technologies that are, after all, intended to be "theirs" in a very real sense of the word (Spiel et al., 2019).

As one step in the formative stages of our process, we conducted an exploratory qualitative study with parents of adolescents with ASD to understand and gain insight into our users' needs and technological preferences. Specific goals included to: (1) identify the aspects of technology parents reported as beneficial for their adolescent and the narratives around why; (2) point out the aspects of technology parents reported as detrimental for their adolescent and the stories around why; and (3) identify the social skills that parents would like their adolescent to develop.

More broadly, this qualitative study allowed us to develop a foundational framework for thinking about adolescent 
users' interests, goals, strengths, and challenges, which then substantively informed the design and development of our new technology-based educational game for teaching ToM and social reasoning skills, as we describe later on in this paper.

A note on terminology: We use "child" or "children" throughout our paper as a general term to refer to individuals from early childhood through adolescence. We use "adolescent" more specifically to refer to older children in the age range of 10-19, following current definitions of adolescence from the APA (American Psychological Association, 2020) and WHO (World Health Organization, 2020).

\section{Children, Technology, and Education}

Over the years, technology use has become ubiquitous in the United States. In 2016, 89\% of households reported having a computer, compared with only $8.2 \%$ in 1984 and $61.8 \%$ in 2003 (File, 2013). Not surprisingly, children are avid consumers of many technologies and engage in browsing the Internet browsing, social media, communicating in online forums, gaming, etc. Children also construct and contribute content to the digital world by designing videos, animations, blogs, and stories (Halverson, \& Sheridan, 2014). These technology-enabled activities, in turn, can extend what it means to socialize in our modern-day world. In fact, many children use technology to make their own content and participate in the growing do-it-yourself movement, a movement involving the production of arts and new technologies (El-Zanfaly, 2015; Knobel \& Lankshear, 2010).

With respect to education, and in light of the considerable time children spend online, educators are interested in how children acquire various forms of technology-related expertise. Digital skills include writing fiction, film editing, and graphic design, across media platforms and diverse online communities. Educators are examining how children interact in these online communities to develop their own innovative constructions, such as games and software programs (Kafai et al., 2012).

This interest in studying children's technological interests and skills extends to educators working with children who have diverse learning needs, such as ASD. Many individuals with ASD tend to have difficulty in initiating social pursuits, such as initiating conversations or activities with peers (Orsmond \& Kuo, 2011). Technology use, however, has shown to be an area of promise in guiding children with ASD to initiate social communication. Researchers, for example, have investigated Autocraft, an online community centered on Minecraft, a popular online multiplayer video game (Ringland et al., 2017). Findings showed how this community supported the development of self-expression and social skills for children with ASD by providing familiarity and structure in a virtual space, while also creating space for children to construct novel ideas and share with others (Ringland et al., 2017). Along similar lines, another team examined the multimodal engagements that three students employed while playing Minecraft (Stone et al., 2019). Atscreen observations and semi-structured interviews revealed that Minecraft provided opportunity for students to engage in conversations, to make requests and give commands, to share information, and to maintain communication with others within the students' physical and virtual worlds (Stone et al., 2019). Although preliminary, these findings are not surprising, in that individuals with ASD tend to prefer predictability, structure, and routine-all features that technologies often provide, in contrast to the unpredictable nature of human interactions (Boucenna et al., 2014). In fact, popular hobbies among children with ASD are frequently sedentary and solitary (Orsmond \& Kuo, 2011).

There has been some research on preferences across technological platforms among children with ASD, and on how they participate in various online communities (Kuo et al., 2014; Martins et al., 2019). However, more research is needed, especially given the accelerating pace at which technology is changing the modes and norms of social interaction across many age groups, settings, and cultures within our society.

\section{Technology Use and Preferences Among Children in General}

Technology is a fundamental part of children's lives today. In the U.S., children between ages eight to twelve spend on average nearly five hours per day using screens for entertainment, and for teenagers between the ages of 13 to 18 , this number increases to over seven hours per day (Rideout, 2019). These figures do not include time spent on screens in school or for homework. Furthermore, social distancing restrictions during the COVID-19 pandemic have increased children's time spent on screens around the world (Guan et al., 2020).

Young children and adolescents often have distinct technology preferences. These preferences are partially driven by stages in cognitive development (Radesky et al., 2015). Children as young as preschoolers and those in the early primary grades, for instance, require more time than adolescents to comprehend media entertainment. When a young child watches a new show, for example, they are attempting to incorporate this content into their limited preexisting framework, which may require additional effort (Flavell et al., 1993). Likewise, young children quickly face cognitive overload, in part due to their developing and smaller working memory capacity (Li et al., 2015).

These cognitive differences can elucidate the preferences observed in the research on children's technology use. For example, one study showed that preschoolers are not 
attracted to faster paced shows (Levine \& Waite, 2000); programs that are slower paced, such as Blue's Clues, are often more attractive. Research studies also find, unsurprising to anyone who has attempted to share a television remote with a small child, that younger children often request to repeatedly watch the same show (Crawley et al., 1999). This is likely because repetition facilitates comprehension, particularly among preschool-aged children (Crawley et al., 1999).

Over time, children's technological preferences shift (Flavell et al., 1993). Children between the ages of seven to 11 prefer faster-paced, adventure packed shows (Valkenburg \& Cantor, 2000). Children in this age range have acquired the working memory capacity to comprehend more challenging information and differentiate reality from fantasy in media (Anderson \& Burns, 1991). Similarly, children in the upperelementary grades become intrigued by real-world events (Wilson, 2008). For instance, by the age of nine-years-old, most children are interested in media content designed for adults (Valkenburg \& Cantor, 2000).

\section{Technology Use and Preferences Among Children with ASD}

Some survey findings have suggested that typically developing (TD) and children with ASD share similar types of technology use (Montes, 2016). Other research, however, has shown that children with ASD play video games significantly more than TD children (Mazurek \& Wenstrup, 2013). Stiller and Mößle (2018) found that across studies, children with ASD preferred action-filled and simulating games.

Similarly, another study examined 172 parent reports about the patterns and impact of technology use in TD children and children with ASD, ranging from 6 to 21 years old. Parents reported that children with ASD had greater compulsive Internet and video game use than their TD peers. Moreover, compared to parents of TD children, parents of children with ASD were significantly more likely to report that technology use had a negative impact on their child (MacMullin et al., 2016).

Likewise, another study investigated television, video game, and social media use in children with ASD compared to their TD siblings (ages 8-18) (Mazurek, \& Wenstrup, 2013). Parents reported on their child's screen-based and extracurricular activities. In comparison to their TD siblings, children with ASD spent more hours daily playing video games and demonstrated significantly higher levels of problematic video game use. However, children with ASD were reported to spend significantly less time using social media or playing socially interactive video games that require players to collaborate and interact.

Indeed, children with ASD do tend to use technology collaboratively less often, although online gaming is more common for young adults with ASD (Kuo et al., 2014).
Research on what children with ASD prefer regarding technology devices and digital content is limited (Stiller \& Mößle, 2018). Nonetheless, some research has suggested that children with ASD prefer animated content, in contrast to realistic, human-like content (Kuo et al., 2014; Schlosser et al., 2019; Shane \& Albert, 2008).

\section{Current Study}

In summary, developing social and ToM interventions for children with ASD will be enhanced by identifying and characterizing the technologies that children with ASD prefer, along with the modes of their interactions and ensuing effects on other aspects of their daily lives with families and friends. While much current research on children with ASD provides valuable information by, for example, measuring the time spent with different kinds of technologies and the devices that children with ASD use (Stiller \& Mößle, 2018), research is also needed to investigate how children with ASD integrate these various forms of technology, including socially rich interactions mediated by technology, into their lives. Morever, these lines of research must be revisited regularly to stay current, as the landscape of technology in children's lives continues to transform at a lightning pace.

Parents often have opportunities to observe their child's technology use and their child's social functioning in many family, peer, and other social settings. As such, we present our findings from the qualitative analysis of 18 semi-structured interviews with parents of adolescents with ASD. (See Study Limitations in our discussion for more on the strengths and limitations of using parents as a source of proxy reports for children with ASD.)

Drawing from the theoretical foundation of a participatory design approach, our team formulated the following research questions to guide our investigation of technological preferences for adolescents with ASD:

1. What aspects of technology do parents report as beneficial for their adolescent and why?

2. What aspects of technology do parents report as detrimental for their adolescent and why?

3. What kinds of social skills do parents believe their adolescent needs to learn?

While parent interviews provide one set of perspectives, and formative research using qualitative methods provides one valuable type of empirical research contribution (van Schalkwyk \& Dewinter, 2020), there is, of course, much more to be done in this area. In particular, the design of educational technologies for children with ASD will benefit from including the perspectives of the children themselves, their teachers, siblings, friends, and other community stakeholders. Moreover, results from this qualitative study, in 
addition to providing concrete ideas for application development in our own work, will serve to suggest new hypotheses and insights that can be addressed in future work, including more in-depth qualitative and quantitative studies. As part of our discussion later in this paper (see section on Study Limitations), we describe specific limitations of this study, including in the context of the prior literature on qualitative and parent-interview-based ASD research, and we also highlight specific directions for future work as informed by our study results.

\section{Methods}

\section{Study Context}

Our parent-interview study was nested within a larger educational technology usability study. This usability study involved two in-person visits by adolescents and their parents that were conducted during the first half of 2019. Visit 1 consisted of having adolescent participants with and without ASD, and between the chronological age of 10 to 17-years-old, come to a laboratory setting to test an existing science-focused educational technology, and Visit 2 had the same participants returning to the laboratory to complete a film-clip-based social reasoning activity. These two activities were selected as part of the formative stages of designing a new educational technology that aims to teach ToM and social reasoning skills by combining concept mapping, as often found in science education, with film-based social skills education. SeeZi et al.'s (2020) study for initial findings from these other portions of the larger study.

During Visit 1 (either during or after the time that the adolescent was engaging with the science learning activity), parents were interviewed about their adolescent's technology use. Adolescents were not present during these interviews. The primary purpose of this paper is to report on these parent interview findings.

\section{Interdisciplinary Study Team}

This exploratory qualitative study involved an interdisciplinary collaboration with experts from four different teams: (1) computer science, (2) pediatrics, psychiatry and behavioral science (hereon after referred to as the clinical team); (3) education and user experience research, and (4) qualitative research. The computer science team consisted of researchers with expertise in artificial intelligence and educational technology, including the investigators and engineers developing the new theory of mind intervention. Researchers on the clinical team assisted in study recruitment and in refining inclusion criteria and other aspects of the study design. The education and user experience research team administered the Visit 1 and Visit 2 sessions, and collaborated with all teams to develop the interview and survey measures for the children and parents. The qualitative research team collaborated with the education and user experience research team to develop the codebook for analysis, assisted in coding, and guided the analysis.

\section{Participants}

This study adhered to the procedures in an approved institutional review board (IRB) protocol. Parents of adolescents with ASD from the United States were recruited to participate in an exploratory study to discuss their adolescent's social skills and experiences with media content. We recruited participating families by using an existing registry currently available to researchers at our institution. This registry guaranteed the credibility of the diagnosis of ASD.

Inclusion criteria for our parent participants were: (1) parent of an adolescent with ASD; (2) adolescent is between ages of 10-17; (3) parent could speak and read English; (4) adolescent's cognitive abilities aligned with their chronological age; and (5) study coordinators conducted phone interviews prior to initial visits to screen parents and ensure that their adolescent met the aforementioned inclusion criteria, and also that their adolescent would be comfortable playing a science game as well as a social-reasoning question/answer activity based on the film clips. Criteria four and five addressed the requirements for adolescents participating in the broader technology usability study. Parent and adolescent participants each received a $\$ 50$ Amazon gift card for each visit, for up to two visits (up to $\$ 100$ for each participant in total).

A total of 22 adolescents with ASD enrolled in the technology usability study during the spring and summer of 2019 and 20 parents (some parents brought multiple children; See Tables 1 and 2). For our parent interview study,

Table 1 Adolescent demographics

\begin{tabular}{lcc}
\hline Characteristic & Sample & $\begin{array}{l}\text { Percent- } \\
\text { age of } \\
\text { sample }\end{array}$ \\
\hline $\begin{array}{l}\text { Autism spectrum disorder } \\
\text { Gender }\end{array}$ & 22 & $100 \%$ \\
$\quad$ Male & 16 & $72.7 \%$ \\
$\quad$ Female & 6 & $27.2 \%$ \\
Race & & \\
$\quad$ Part or Full Hispanic & 5 & $22.7 \%$ \\
Part or Full White & 10 & $45.5 \%$ \\
African American & 1 & $4.6 \%$ \\
Other or not specified & 3 & $13.6 \%$ \\
Unreported & 3 & $13.6 \%$ \\
\hline
\end{tabular}


Table 2 Parent demographics

\begin{tabular}{lll}
\hline Characteristic & Sample & Percentage \\
\hline $\begin{array}{l}\text { Parent of adolescent with autism } \\
\text { spectrum disorder }\end{array}$ & 18 & $90 \%$ \\
Gender & & \\
Male & 3 & $16.6 \%$ \\
Female & 15 & $83.3 \%$ \\
\hline
\end{tabular}

Table 3 Ownership of technology ( $n=15$ reported this information)

\begin{tabular}{lll}
\hline Type of technology & Sample & Percentage \\
\hline Laptop and/or computer/desktop & 12 & $80 \%$ \\
Television & 11 & $73 \%$ \\
Cell phone & 8 & $53 \%$ \\
Tablet & 6 & $40 \%$ \\
Video game console & 9 & $60 \%$ \\
\hline
\end{tabular}

we planned to interview the 20 parents, but two of the parent interviews could not be administered due to scheduling difficulties. Thus, this paper reports on the qualitative analysis of 18 interviews from parents of adolescents with ASD. None of the 18 parent participants were partners, shared a household, or were parents to the same adolescent. The 22 adolescents with ASD ranged in age from 11 to 17, with a mean age of 14. (Female participating adolescents ranged in age from 11 to 16 , with mean age 13 , and male participating adolescents ranged in age from 10 to 17 , with mean age 14) Of the 15 adolescent participants who did provide additional demographic information, 5 identified as being full or part Hispanic, 10 identified as being full or part White, 1 identified as being Black, and 3 identified as full or part "other" (not specified). We did not collect any explicit measures of social skills or level of difficulties from the adolescent participants, beyond screening participants per our inclusion criteria, as described above.

We did not collect explicit measures of family SES, but we did ask adolescent participants about their ownership of various electronic devices (where ownership included their own personal device as well as devices shared within the household, except for cell phones which we report on here specifically for their own devices; See Table 3). Of the 15 adolescent participants who provided this information, 12 reported owning a laptop and/or desktop computer; 11 reported owning a television; 8 reported owning their own cell phone; 6 reported owning a tablet; and 9 reported owning a video game console.

\section{Study Procedure}

First, the researcher provided parent participants with information about this study, including their rights as participants and that participation was voluntary and they could stop at any point. The researcher then asked if participants wanted to proceed and whether they consented to be audio recorded during the interview.

All interviews were audio recorded and lasted from 30 to $60 \mathrm{~min}$. The audio recordings were transcribed using an IRB-approved transcription service (rev.com). To maintain a sense of ease and informality, the researcher did not take notes during the interviews. Audio files and transcripts were stored on a secure server maintained by the authors' institution and were accessible only by members of our study team. Each interview was assigned a unique participant ID number, which was used to create a unique file name for each transcript. The research team removed all identifying information from the transcripts. These de-identified transcripts were each put in an Excel file then which was used for qualitative coding and analysis.

Note that the participant ID numbers included in this article have been re-randomized to further protect participant privacy. We include ID numbers throughout this article to help convey how our qualitative results are drawn from across our participant sample.

\section{Interview Guide}

A semi-structured interview guide was used and the questions were informed by prior qualitative empirical studies in this area of study (Abirached et al., 2012; Kuo et al., 2014; Whyte et al., 2015). Specifically, the interview guide for parents included three sections. First, the researcher asked parents about their adolescent's screen time and technology use, specifically their adolescent's favorite shows, video games, and social media. Additionally, parents were asked to explain why these technology activities were interesting to their adolescent. Second, parents were asked to describe how technology use affected their adolescent's behavior and mood. Finally, parents were asked to identify the social skills they wanted their adolescent to develop.

\section{Data Analysis}

Qualitative coding occurred in two phases: (1) individual quotes were isolated in the transcripts; and (2) a hierarchical coding system was developed based on the interview schedule and a preliminary review of the transcripts. Definitions were written for each category (see Supplementary Materials).

Major categories obtained through the coding process were: (1) Time spent on daily electronic use; (2) Types of 
technology used; (3) Technology-based activities in which the adolescent engages; (4) Environment in which technology is used; (5) Mood and behavior; and (6) Potential computer-based interventions. Each major category was subdivided, and the subcategories were expanded to capture additional thematic detail.

Coding was conducted by two trained coders, the first and third authors. They individually coded four selected transcripts, and then they compared each coded transcript to resolve any discrepancies. The remaining transcripts were divided and coded independently.

As a further note on the reliability of our coding methods, we viewed coding as less of a process of classification and more as a way to use an established and agreed upon language - that is, the coding system - to capture the meaning of the quotes. This is why we approached establishing reliability as a process rather than a statistic. Instead of double coding and reconciling all transcripts, which would have been too resource intensive for the scope of our study, we double coded and reconciled four out of our 18 transcripts (i.e. 20\%), and we did not proceed to allowing a single coder to code a transcript until we felt confident that there was a shared understanding of the coding categories and how to use them.

After coding, the coded transcripts were combined into a single file and sorted by category. The transcripts, quotations, and codes were managed by Microsoft Excel 2016 and SPSS version 26.0. Analysis was performed using an Excel file with quotes sorted by coding category.

An iterative inductive-deductive approach was used to analyze the sorted coded quotes (Fereday \& MuirChochrane, 2006). Deductively, we were guided by social constructivist theory, in that we explored how adolescents with ASD actively used technologies and sometimes coconstructed knowledge by using technologies within different kinds of social contexts and communities. Social constructivist theory is based on the work of Vygotsky and seeks to understand how children develop (or construct) a cognitive understanding of the world through interaction with others in a cultural context (Vygotsky, 1978). We see interactions with parents, peers, and technology as drivers of cognitive development and vice versa, which also aligns with more specific theoretical and empirical work on the interplays between theory of mind and other cognitive abilities in ASD and social learning/interaction (Hughes $\&$ Leekam, 2004). This perspective helped us develop a coding system that seeks to capture adolescents' interactions with people and technology as influencing, and being influenced by, their developing a theory of mind and social skills. Inductively, we used the coded quotes to identify themes and relationships between themes.

More specifically, our iterative inductive/deductive process involved reading the quotes within categories, beginning to make connections between categories, organizing our understanding of the material, and iterating on these. Our analytical approach drew from latent content analysis, in that we aimed to discover the underlying meanings and stories behind the various accounts parents reported that technology had on their adolescent's behavior and mood (Hsieh \& Shannon, 2005).

We chose this analysis method because this is a foundational, generative study aimed at qualitatively uncovering key themes that could inform the design and development of our game. Our focus is on discovery of underlying meanings in the data rather than trying to provide summative tallies or frequency estimates of observed themes (though these kinds of summative approaches may certainly be useful in future work that is geared more to specific hypotheses).

We identified two major themes most relevant to our research questions: (1) parents' accounts of the impact technology use had on their adolescent's behavior and mood; and (2) specific social skills they wanted their adolescent to learn in order for their adolescent to more effectively navigate social situations and cultivate relationships.

\section{Results}

\section{Behavior and Mood}

Parents described both positive and negative effects of technology use on their adolescent's behavior and mood. Many parents expressed how media was a self-soothing activity for their adolescent, especially after a long day. Others expressed that technology sometimes caused dysregulatory behaviors, particularly if a game or device did not go their adolescent's way. Regardless of the positive and negative accounts, the majority of parents described feeling conflicted about their adolescent's technology use. Some parents expressed how technology could be calming and was often a creative environment for their adolescent to learn new skills. However, these same parents described that their adolescent often lost touch with real-world social communication due to using their devices too much and/or due to growing frustrated when a technological activity did not go as desired (e.g., losing a game). Next, we describe these accounts in detail.

\section{Positive Accounts}

The majority of parents described some types of media that had positive effects on their adolescent's behavior and mood. Several parents described how playing games on devices such as the iPad would induce calmness and relaxation for their adolescent. Using technology, in one's parent's words, often functioned as a way for her son to "decompress" after 
a long day (participant ID 1, hereon after referred to as PID). This parent later described how "watching television" would "kind of just like take you out of yourself," in that watching TV became a way for her son to escape the present reality and self-soothe (PID 1). Another parent discussed how playing games not only "relaxes" her son but "pumps up his self-esteem," because "he's good at it" (PID 20).

In addition to inducing calmness, many parents described how their adolescent used technology to develop technical skills and learn about topics of interest, such as issues related to social justice and even their own ASD diagnosis. One parent described how her son played the games to "get into the software system and look at the guts and the inner workings and components and setup files" (PID 23). In other words, her son wanted to learn how the games worked on the backend and developed software skills, such that his mom described how the software "... spoke his language. He understood it immediately and within two months he said, Okay I'm done with this. Now I want this other advanced one" (PID 23).

Other parents discussed how their adolescents would play games that enhanced personal agency, in that the players had the creative license to create characters, a story, and so forth. Minecraft was one game that many parents identified as a preferred game. One mother elaborated on the appeal of Minecraft for her daughter:

"Anything for her that she can explore in a safe environment and a very controlled environment is perfect. She never plays hard mode Minecraft. She never does anything where there's a survivor ability to it. It's all about what can I do to this world? How can I mold this world the way I want it to be? So it's always creative, and it's always with full permissions that she can have, because then she has full control over it." (PID 9)

Here, the parent explains how having the "control" to "mold" and shape the game-like world in a manner that her daughter prefers feels "safe." This safety, in turn, opens the door for her daughter to exercise and cultivate her creative skills via the gaming experience.

\section{Negative Accounts}

Most parents described a number of negative behavioral and emotional impacts technology has on their adolescent. Several parents discussed how technology can frustrate their adolescent, particularly during technical difficulties and/or if a game did not proceed in a manner their adolescent desired. One parent described engaging with technology in general as "an opportunity" for his son's tendencies to have a "melt down" to emerge because if "he's trying to play a game on the computer and the computer freezes or a sound isn't working, any of those things where something isn't working the way it's supposed to, those are triggers for his more explosive frustration" (PID 25). Other parents described how if the games did not go their adolescent's way, that is, if their adolescent lost a game, they became unhappy. "Basically, difficulty he was having getting through a level. And when [the] game failed or whatever, sometimes he'd get upset...Definitely not happy with the situation" (PID 9). In this sense, if the game became too difficult, the game would then often disrupt the enjoyment and overall flow in the gaming experience, such that, in this mother's words, her son would be left feeling "definitely not happy" (PID 9).

\section{Mixed Accounts}

Many parents were uncertain whether their adolescent's technology use had a net positive or negative impact on their adolescent's behavior and mood. On the one hand, technology seemed to be a go-to, self-soothing strategy for many adolescents in our sample. On the other hand, technology seemed to function as a way for the adolescents to escape from social situations, or the adolescents would so much time on the screen that they seemed to forget how to socially behave. One parent described how "I don't know if it's that maybe that length of time without human interaction, she just forgets that you can't act like a jerk" (PID 28). Another parent described how she would encourage her son to "be social even while he's at home," but that he preferred to use technology, specifically "by himself" (PID 7). She then later described that after being on the screen for so long, her son would grow "foggy" and start "looking up one of his things [specific interests, such as car registration numbers]" and not come for "dinner" or start another family activity (PID 7).

Some adolescents in our sample relied on electronic communication to communicate with peers and family members. Although communicating digitally removed the adolescent from experiencing real-world social interaction, parents described some positive benefits. One parent discussed how her daughter could "keep in touch with her sister that we don't get to see as often," or contact her mom if she was "being bullied" (PID 4). At the same time, however, this same parent discussed the drawbacks: "The drawbacks are you never get to see their faces because she's always down in the phone... There's new things going up. There's people you can't see that you... because your head's in your phone" (PID 4). In this sense, technology use sometimes resulted in adolescents socially withdrawing from their real and live physical and social environments, in that they would become immersed and absorbed in whatever activity they were engaging in via their devices. Nevertheless, there was a fine line between 
when parents reported that their adolescents used too much technology and became detached from present social interactions and when their adolescents used technologies to fruitfully sustain relationships with peers and family members.

\section{Parents' Suggestions on Targeting Social Skills for Interventions}

There were several social skills that parents reported that they would want their adolescent to develop in a technologybased intervention (See Tables 4 and 5). Although many of these skills overlapped, we identified two clear distinctions in how parents described the skills.

Skills like impulse control, emotion regulation, and selfconfidence are related to self-regulation, which is integral to social interaction. Skills like understanding others' nonverbal cues, engaging fully in social interaction and communication, understanding various contexts in relationships, and building relationships (e.g., friendships) over time are all related to interpersonal sensitivity. Next, we describe these subcategories-self-regulation and interpersonal sensitivity-in further detail.

\section{Self-Regulatory Skills}

Many parents identified that their adolescent struggled with regulating their behaviors and emotions in social situations. One parent described challenges with impulse control, in that their adolescent did not understand that it was inappropriate to "be very vocal" and express to the whole classroom that they did not want to sit next to a particular peer (PID 23). Likewise, another parent shared how her daughter could be "...extremely aggressive, especially with her brother" and expressed her aggression in ways that were "bossy and rude," such that other peers felt frightened (PID 28). On a similar note, one parent reflected on her daughter's difficulties with emotion regulation and her challenge in internally feeling ready to start "screaming and running around" and not having the tools to regulate effectively (PID 9). Similarly, another parent described her son's fear of knocking on the door or hearing the door be knocked, and shared a time when her son was

Table 4 Summary of parents' target self-regulatory skills

\begin{tabular}{|c|c|c|}
\hline Social skill & Description & Example \\
\hline Impulse control & $\begin{array}{l}\text { Being able to pause and think before acting; controlling } \\
\text { impulsive behaviors, such as inhibiting the impulse to say } \\
\text { inappropriate or rude remarks }\end{array}$ & $\begin{array}{l}\text { "Sometimes she just doesn't like who she's sitting next to, } \\
\text { and she'll be very vocal about it and stand up in her chair } \\
\text { and say, No, I refuse to do classwork with this particular } \\
\text { person." (PID 23) } \\
\text { "I would love for her to have more of a stop before you act. } \\
\text { When I go back through and think of all of the different } \\
\text { times where she's become escalated to any extent, if she } \\
\text { would just stop before she acted or said something and } \\
\text { took her time to breathe and actually do the "Okay, let me } \\
\text { think about what I'm about to say or what I'm about to } \\
\text { do," that would help out tremendously." (PID 9) }\end{array}$ \\
\hline Emotion regulation & $\begin{array}{l}\text { Identifying one's own emotions, whether they are positive } \\
\text { or negative, and being able to regulate one's emotions in } \\
\text { various situations, especially in unknown circumstances, } \\
\text { disruptive situations, etc.; understanding how to manage } \\
\text { emotions during transitional periods }\end{array}$ & $\begin{array}{l}\text { "She is very aware of her own scale of a } 0 \text { through } 10 \text { on } \\
\text { where she's at...Externally she can be on a } 1 \text { or a } 2 \text {, but } \\
\text { internally she's about ready to start throwing things and } \\
\text { yelling and screaming and running around." (PID 9) } \\
\text { "[Name] tried to stand up to her, and she assaulted him } \\
\text { basically, and she scratched him so hard, that's what } \\
\text { caused the bruising. Well, and then she punched it. And } \\
\text { he said she told him, "If you tell, I'm going to do it again, } \\
\text { and I'll make it worse. If you make my life worse, I'm } \\
\text { going to make yours even worse." So he was afraid. She } \\
\text { kind of terrorizes him, and it's like sibling rivalry on } \\
\text { steroids, if that makes sense." (PID 28) }\end{array}$ \\
\hline Self-confidence & $\begin{array}{l}\text { Having trust and positive beliefs about one's abilities, quali- } \\
\text { ties, etc }\end{array}$ & $\begin{array}{l}\text { “It's hard to get him to advocate for himself. He doesn't } \\
\text { realize what's going on.” (PID 23) } \\
\text { “I feel a little more self-confidence, I think. He has confi- } \\
\text { dence in what he does all by himself, but in the situation } \\
\text { around people, if he's called on to do something, he'll do } \\
\text { it. If he can help somebody else, he gladly will. He loves } \\
\text { helping other people but it's hard to get him to advocate } \\
\text { for himself. He doesn't realize what's going on.” (PID 3) }\end{array}$ \\
\hline
\end{tabular}

Participant IDs have been re-randomized for this article to protect privacy 


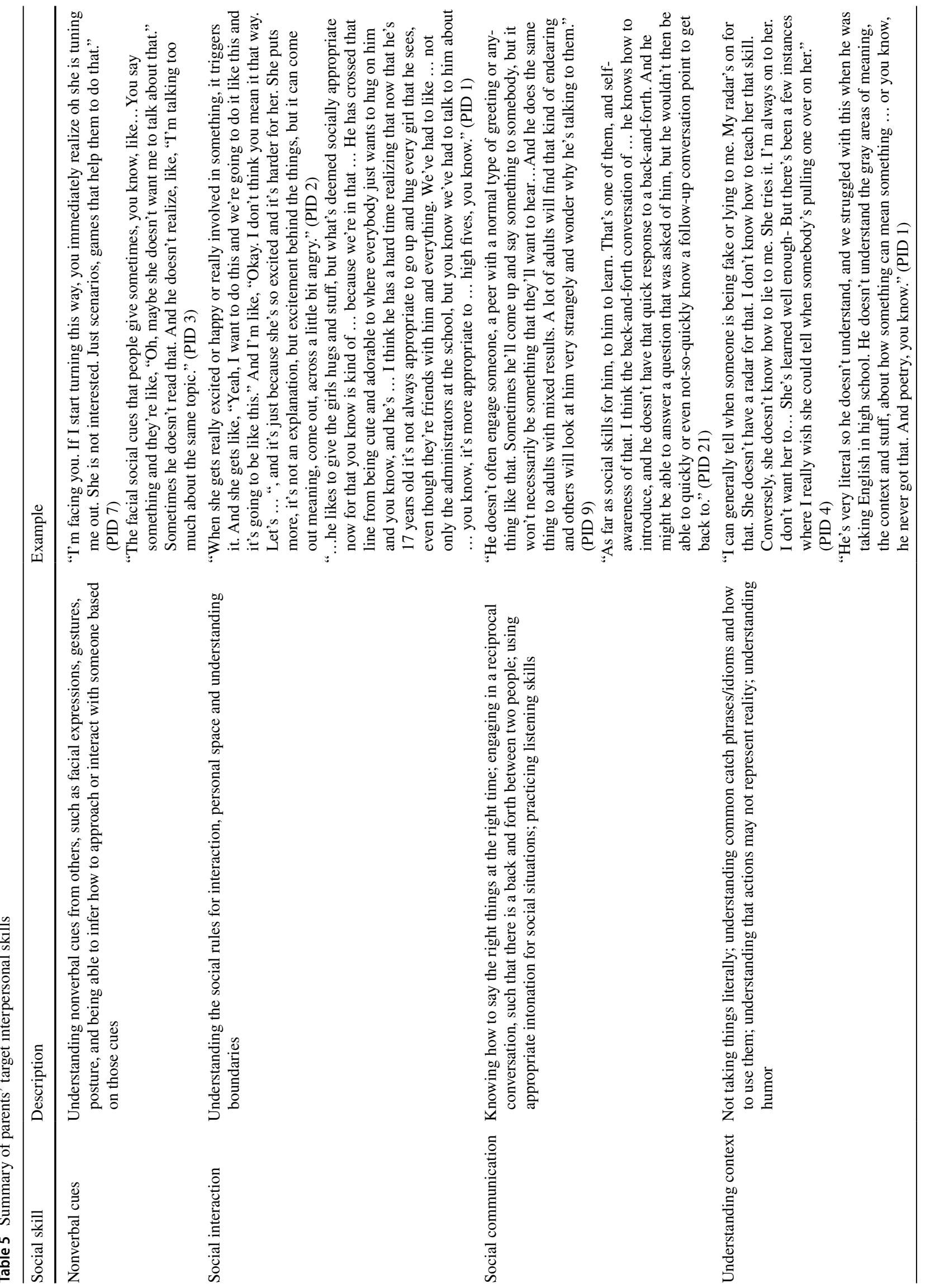


found "...in the corner of [her] office walking back and forth and crying for $20 \mathrm{~min}$ before anybody realized that" (PID 8). She then identified that it would have been helpful for him to understand how to cope with events, such as when someone knocks on his door, or when he is invited to try a new activity. In other words, this parent expressed wanting her son to learn how to manage "fear" in a more emotionally effective manner, such that he would not be "crying for 20 min" in a "corner" where he would be difficult to find. Many parents also shared how their adolescent struggled to "advocate" for themselves and have the selfconfidence they needed to succeed (PID 23).

\section{Interpersonal Sensitivity}

Parents described wanting their adolescents to cultivate greater interpersonal sensitivity, the ability to accurately judge others' thoughts, feelings and behaviors using verbal, non-verbal, and contextual cues (Hall \& Bernieri, 2001). As Table 5 shows, many parents shared how their adolescent often did not readily understand how to read nonverbal cues and specifically how to position one's body within a social context. Likewise, we observed the interrelationships between parents' accounts of their adolescent's limited self-regulatory skills and how this limitation negatively impacted social interaction. One parent, for instance, described how her daughter sometimes would feel really "happy" or "excited" and then socially interact with others in ways that "came across as angry" (PID 2). On a similar note, social communication was another area for growth. One parent described how his son did not know how to "greet" adults and especially peers (PID 9). We attributed this difficulty to her son's anxiety, specifically with regards to not knowing how to navigate unknown social situations and thus novel emotions effectively.

We also noticed an interesting relationship between parents' accounts of their adolescent's abilities to understand various social contexts and to develop positive relationships. For example, one parent described how her daughter did not know when others were lying, or when "somebody's pulling one over on her" (PID 4). She described a situation where her daughter had thought she made several new friends at school when in reality they weren't genuinely interested in being her friend. The mother implied that her daughter could not understand deception, deception that could be embedded within a larger social context of navigating social dynamics at the junior high school level. Consequently, the daughter might not have understood when someone genuinely wanted to be her friend, rather than being confronted with a person who had competing motives, motives that might not lead to healthy relationships. With regards to building relationships, another 
parent discussed how although her son had friends who came to the "house" and were "always really nice," her son did not "really have a friend" who he could depend on (PID 3). Her son might not have understood the social context as to why his friends were "really nice" nor had the tools to develop deeper, meaningful friendships.

\section{Discussion}

This study investigates, through qualitative analysis of parent interviews, how adolescents with ASD use technology and the effects that technology has on their behavior and mood. We also identified social skills parents would like their adolescents to learn via potential technology-based interventions. Corroborating prior literature, we found that the patterns of technology use and activities that most adolescents with ASD in our sample gravitated toward consistent mainly of online applications, video games, and watching video clips (Kuo et al., 2014; Mazurek \& Wenstrup, 2013; Stiller \& Mößle, 2018).

Corroborating previous research, parents also shared how technology, specifically games like Minecraft, supported the development of their adolescent's creativity and self-expression by providing familiarity and structure in a virtual environment, while also creating a space for their adolescent to construct novel ideas (Ringland et al., 2017). Furthermore, we found that parents reported positive effects of technology on their adolescent's behavior and mood, in that engaging with some technologies provided ways for many adolescents in our sample to remove themselves from stressful situations (e.g., a long day at school) and restore positive emotions (Boucenna et al., 2014; Orsmond \& Kuo, 2011). At the same time, however, and aligned with prior research, there were instances of parents' conflicting opinions on how technology impacted their adolescent's behavior and mood, specifically when a game did not go the adolescent's way and/or when the adolescent was immersed in a technologically centered activity and did not want to engage in human interaction (MacMullin et al., 2016).

This study also explored the social skills parents prioritized for their adolescent to develop. Parents wanted their adolescent (1) to improve their self-regulatory skills, and (2) to cultivate greater interpersonal sensitivity in various social contexts.

Interestingly enough, it has been well-documented in the scientific literature that self-regulation and social sensitivity are interrelated. In other words, people need to have sufficient self-awareness of their own behavior in order to be able to measure it against typical social norms (Heatherton, 2011). Challenges in self-regulation are often related to poor relationships, decreased job success, suboptimal mental health, and a whole host of negative outcomes, whereas enhanced self-regulation is often related to the inverse (Heatherton \& Wagner, 2011).

Many parents in our study identified this relationship and described how their adolescent needed to first improve on identifying their own emotions and behaviors, that is, self-awareness and self-regulation, before they could develop positive relationships with others. Such a finding is consistent with prior literature, in that people need to be able to well-regulated in order to understand how others react to their behavior and predict how others might respond to them (Heatherton, 2011).

\section{Study Limitations}

This study provides useful information towards understanding parents' observations about the effects that technology has on their adolescent's behavior and mood, and about the kinds of social skills that parents would like to see their adolescent develop. Nevertheless, certain methodological factors may limit the generalizability of these findings.

First, the small sample $(n=18)$, although typical of qualitative studies, makes it difficult to investigate how differences in social-economic status, age, gender, race, ethnicity, or other demographic or cultural factors might affect these findings. For example, an individual's own cognitive and social capabilities, as well as their relationships to technology and to other people, must evolve significantly during adolescence, and so additional research is needed to situate findings in the contexts of age and development within adolescence. In addition, gender also exerts significant influence both on adolescence and social interaction in general as well as within ASD specifically, for instance as with the female autism phenotype and its influence on a person's social development (Bargiela et al., 2016). In future work, larger, more quantitative studies will be important for describing differences between males and females and/or between different age groups.

Second, our qualitative study of parents was carried out within the context of a broader project for developing a new theory of mind (ToM) and social reasoning game aimed at adolescents with ASD who have challenges or need support in regards to social skills and communication and who need only limited support in reading comprehension and/or other academic skills. While adolescents within ASD have been specifically called out as needing more research in social skills interventions (Reichow \& Volkmar, 2010), our study and findings do not address other subgroups in ASD (e.g., adolescents having lower levels of independence or everyday functioning skills) who may use technology differently and likely face social challenges that vary in kind and degree from those discussed by parents in our sample. We also do not address similarities or differences in parent perspectives 
on technology use by their adolescent with ASD in comparison to any typically developing siblings in the household, i.e., this study does not speak to potential differences in technology use, or parent perceptions of it, between typically developing children and children with ASD.

Third, this paper reports data collected from parents and not from the adolescents with ASD themselves, each approach having its own strengths and limitations. Prior work has argued that interview data from children with ASD, specifically qualitative approaches using interpretative phenomenological analysis, can better ensure a foundation of equality between the participant and researcher, thus elucidating the experiences of individuals with ASD. In the context of our study, such an approach could have extended opportunities for users with ASD to represent their needs and goals when using technology (MacLeod, 2019). Interviewing close proxies to users with ASD, such as parents, is increasingly acknowledged in the literature as contributing somewhat different but complementary forms of information. With parent interviews, as we have used here, there is the possibility that parents' accounts may not fully reflect their child's typical media use, patterns, and effects on behavior and mood (Kuo et al., 2014). For example, one qualitative study reported on the tensions between typically developing adolescents and their parents around technology use and found that parents often underestimate the quantities and varieties of social media that their adolescents were using (Blackwell et al., 2016). On the other hand, a surveybased study of parents of children with ASD speculated that different parents may over- or under-estimate their child's technology use (Laurie et al., 2019). Taken together, parents' perspectives may serve to triangulate self-reported findings from children, especially in light of preliminary research showing that sometimes discrepancies in descriptions of a child's social perceptions and social skills can occur between parents and children with ASD (Lerner et al., 2012; McMahon, \& Solomon, 2015; Rankin et al., 2016). In future work, the accuracy of parent perspectives about their children in studies like ours could be better assessed, for example, by asking parents how closely (or not) they monitor their child's technology use, and also by collecting data from parents and children in order to better compare the different perspectives.

\section{Key Takeaways for Technology Design}

Our findings have informed the development of our social skills and ToM educational game in several ways. We present our key takeaways and ensuing design decisions here, to help inform other research and development efforts creating educational technologies for adolescents with ASD.

\section{Increasing Focus on Topics of Complex Social Context}

Our original game concept focused primarily on promoting adolescents' comprehension of social and ToM concepts appearing in film clips, i.e., building our user's base of knowledge and perceptual/reasoning skills in topics related to interpersonal sensitivity. In particular, one of the core activities of our game asks users to watch short movie clips in the application and then answer quiz questions, questions that probe their understanding of the social content in the clip and hopefully improve comprehension of and internalization of the target social skills.

Given that our users might need limited support in academic areas and require more extensive support around social and communication skills, our conversations with parents highlighted the challenges that adolescents with ASD face as they interact with their neurotypical peers, who are engaging in increasingly sophisticated modes of social interaction that users with ASD can have trouble comprehending. Taking parents' comments into account, we are paying particular attention in selecting clips that touch upon topics of complex social context, building friendships, and effectively dealing with negative emotions. We have also developed a library of questions starting from easy (e.g., questions assessing the literal sequence of events) to progressively challenging (e.g., questions measuring the user's understanding of a character's emotion and motive for behaving one way toward another character).

\section{Incorporating Activities for Self-Regulation into the Game}

Self-regulation, cited so heavily in our interviews with parents of adolescents with ASD, did not originally appear anywhere in our educational game for teaching social and ToM skills. In particular, parents discussed self-regulation as important both within the context of social interactions, which our game (a single-player game) cannot readily train, as well as within the context of interactions with technology, which our game can and does embody. Therefore, we decided to incorporate new features that explicitly aim to track and improve students' self-awareness of emotion and self-regulation.

For example, we plan to include survey prompts at the start of each game session that include questions about the user's current emotional state, and, for example, how their day has gone so far. These surveys are intended not only as part of our research data collection but also to get users to pause, even if just for a moment, to reflect on how they are feeling before each session. The game also now has a new "mindfulness arcade" area that players can visit if they feel frustrated or stuck. In this area, users will have options to learn about and participate in brief self-soothing activities before resuming their game-play (e.g., breathing 
exercises, interactive coloring, physical stretching), to give them explicit training and support in small practices for self-regulation.

\section{Additional Takeaways}

There were several takeaways that we are not able to implement in our own game but that we present here as part of our discussion and consideration for future and related work. Our parent interview findings here are consistent with prior literature in that providing players with a creative story and agency are often-discussed routes for sustaining engagement and building confidence (Halverson, \& Sheridan, 2014). Likewise, a technological intervention that fosters an online community and ways for players with ASD to share their ideas, experiences, and co-construct knowledge may also enhance engagement, in addition to facilitate the development of understanding context and building relationshipstwo key skills many parents identified as areas for growth (Kafai et al., 2012; Vygotsky, 1978). Designing or leveraging technologies that invite players to delve into a virtual world and have the agency to create a story, characters, and so forth in the gameplay experience promises to be an area of opportunity for future educational technologies for adolescents with ASD.

\section{Implications and Future Directions}

Future research may include interviews with siblings, friends, teachers, and individuals with ASD themselves to understand the contextual circumstances that lead users with ASD to become immersed in a technology-centered activity, including positive, engaged immersion as well as the negative aspects of immersion that parents in our study referenced. Future research is also needed to understand the degree to which the kinds of social skills learned from technological interventions, such as games and applications, can be generalized to the physical, real world and in-person social interactions of adolescents with ASD.

Supplementary Information The online version contains supplementary material available at https://doi.org/10.1007/s10803-021-05315-y.

Acknowledgments We would like to thank all of the parents and adolescents who took part in our study. We also thank the reviewers of this paper for their constructive comments.

Author Contributions RNR and MK planned this exploratory study. A5-A8 helped design specific recruitment and study procedures, and also contributed to participant recruitment and screening. A1 conducted the interviews. A1 and A3 coded the transcripts. A1-A4 analyzed the data. All authors discussed the initial study design and interpretation of the data, and read and approved the final manuscript.
Funding The research reported here was supported by the Institute of Education Sciences, U.S. Department of Education, through the Institution of Educational Sciences. The opinions expressed are those of the authors and do not represent views of the Institute or the U.S. Department of Education.

\section{Declarations}

Conflict of interest The authors declare that they have no conflict of interest.

Ethical Approval The protocols used in this research study were approved by the ethics committee at Vanderbilt University.

Informed Consent Informed consent was obtained from all individual participants included in the study.

\section{References}

Abirached, B., Zhang, Y., \& Park, J. H. (2012). Understanding user needs for serious games for teaching children with autism spectrum disorders emotions. EdMedia Innovate Learning (pp. 1054-1063). Association for the Advancement of Computing in Education (AACE).

American Psychological Association. (2020). Adolescence. APA Dictionary of Psychology. Accessed online on January 10, 2021. https://dictionary.apa.org/adolescence

Anderson, D. R., \& Burns, J. (1991). Paying attention to television (pp. 3-25). Responding to the screen: Reception and reaction processes.

Bargiela, S., Steward, R., \& Mandy, W. (2016). The experiences of latediagnosed women with autism spectrum conditions: An investigation of the female autism phenotype. Journal of Autism and Developmental Disorders, 46(10), 3281-3294.

Blackwell, L., Gardiner, E., \& Schoenebeck, S. (2016). Managing expectations: Technology tensions among parents and teens. In Proceedings of the 19th ACM Conference on Computer-Supported Cooperative Work \& Social Computing (pp. 1390-1401)

Boucenna, S., Narzisi, A., Tilmont, E., Muratori, F., Pioggia, G., Cohen, D., \& Chetouani, M. (2014). Interactive technologies for autistic children: A review. Cognitive Computation, 6(4), 722740. https://doi.org/10.1007/s12559-014-9276-x

Crawley, A. M., Anderson, D. R., Wilder, A., Williams, M., \& Santomero, A. (1999). Effects of repeated exposures to a single episode of the television program Blue's Clues on the viewing behaviors and comprehension of preschool children. Journal of Educational Psychology, 91(4), 630. https://doi.org/10.1037// 0022-0663.91.4.630

Dolan, J. E. (2016). Splicing the divide: A review of research on the evolving digital divide among $\mathrm{K}-12$ students. Journal of Research on Technology in Education, 48(1), 16-37.

Durlak, J. A. (2015). Studying program implementation is not easy but it is essential. Prevention Science, 16(8), 1123-1127. https://doi. org/10.1007/s11121-015-0606-3

El-Zanfaly, D. (2015). [I3] Imitation, Iteration and Improvisation: Embodied interaction in making and learning. Design Studies, 41, 79-109. https://doi.org/10.1016/j.destud.2015.09.002

Fereday, J., \& Muir-Cochrane, E. (2006). Demonstrating rigor using thematic analysis: A hybrid approach of inductive and deductive coding and theme development. International Journal of Qualitative Methods, 5, 80-92. 
File, T. (2013). Computer and internet use in the United States (pp. 20-568). Current population survey reports.

Flavell, J. H., Miller, P. H., \& Miller, S. (1993). Cognitive development. Prentice-Hall.

Fletcher-Watson, S., De Jaegher, H., van Dijk, J., Frauenberger, C., Magnée, M., \& Ye, J. (2018). Diversity computing. ACM Interactions, 25(5), 28-33.

Fletcher-Watson, S., McConnell, F., Manola, E., \& McConachie, H. (2014). Interventions based on the theory of mind cognitive model for autism spectrum disorder (ASD). Cochrane Database of Systematic Reviews. https://doi.org/10.1002/14651858.cd008 785.pub2

Frith, U. (1994). Autism and theory of mind in everyday life. Social Development, 3(2), 108-124. https://doi.org/10.1111/j.1467-9507. 1994.tb00031.x

Guan, H., Okely, A. D., Aguilar-Farias, N., Del Pozo, C. B., Draper, C. E., El Hamdouchi, A., Florindo, A. A., Jáuregui, A., Katzmarzyk, P. T., Kontsevaya, A., Löf, M., Park, W., Reilly, J. J., Sharma, D., Tremblay, M. S., \& Veldman, S. L. C. (2020). Promoting healthy movement behaviours among children during the COVID-19 pandemic. The Lancet Child \& Adolescent Health, 4(6), 416-418. https://doi.org/10.1016/s2352-4642(20)30131-0

Hall, J. A., \& Bernieri, F. J. (2001). Interpersonal sensitivity: Theory and measurement. Lawrence Erlbaum Associates Inc.

Halverson, E. R., \& Sheridan, K. (2014). The maker movement in education. Harvard Educational Review, 84(4), 495-504. https:// doi.org/10.17763/haer.84.4.34j1g68140382063

Heatherton, T. F. (2011). Neuroscience of self and self-regulation. Annual Review of Psychology, 62, 363-390. https://doi.org/10. 1146/annurev.psych.121208.131616

Heatherton, T. F., \& Wagner, D. D. (2011). Cognitive neuroscience of self-regulation failure. Trends in Cognitive Sciences, 15(3), 132-139. https://doi.org/10.1016/j.tics.2010.12.005

Hsieh, H.-F., \& Shannon, S. E. (2005). Three approaches to qualitative content analysis. Qualitative Health Research, 15(9), 1277-1288. https://doi.org/10.1177/1049732305276687

Hughes, C., \& Leekam, S. (2004). What are the links between theory of mind and social relations? Review, reflections and new directions for studies of typical and atypical development. Social Development, 13(4), 590-619.

Kafai, Y. B., Fields, D. A., Roque, R., Burke, W. Q., \& MonroyHernandez, A. (2012). Collaborative agency in youth online and offline creative production in Scratch. Research and Practice in Technology Enhanced, 7(2), 63-87.

Knobel, M., \& Lankshear, C. (Eds.). (2010). DIY media: Creating, sharing and learning with new technologies (Vol. 44). Peter Lang.

Kuo, M. H., Orsmond, G. I., Coster, W. J., \& Cohn, E. S. (2014). Media use among adolescents with autism spectrum disorder. Autism, 18(8), 914-923. https://doi.org/10.1177/1362361313497832

Laurie, M. H., Warreyn, P., Uriarte, B. V., Boonen, C., \& FletcherWatson, S. (2019). An international survey of parental attitudes to technology use by their autistic children at home. Journal of Autism and Developmental Disorders, 49(4), 1517-1530. https:// doi.org/10.1007/s10803-018-3798-0

Lerner, M. D., Calhoun, C. D., Mikami, A. Y., \& De Los Reyes, A. (2012). Understanding parent-child social informant discrepancy in youth with high functioning autism spectrum disorders. Journal of Autism and Developmental Disorders, 42(12), 2680-2692. https://doi.org/10.1007/s10803-012-1525-9

Levine, L. E., \& Waite, B. M. (2000). Television viewing and attentional abilities in fourth and fifth grade children. Journal of Applied Developmental Psychology, 21(6), 667-679. https://doi. org/10.1016/s0193-3973(00)00060-5

Li, H., Boguszewski, K., \& Lillard, A. S. (2015). Can that really happen? Children's knowledge about the reality status of fantastical events in television. Journal of Experimental Child Psychology, 139, 99-114. https://doi.org/10.1016/j.jecp.2015.05.007

MacLeod, A. (2019). Interpretative phenomenological analysis (IPA) as a tool for participatory research within critical autism studies: A systematic review. Research in Autism Spectrum Disorders, 64, 49-62. https://doi.org/10.1016/j.rasd.2019.04.005

MacMullin, J. A., Lunsky, Y., \& Weiss, J. A. (2016). Plugged in: Electronics use in youth and young adults with autism spectrum disorder. Autism, 20(1), 45-54. https://doi.org/10.1177/1362361314 566047

Martins, N., King, A., \& Beights, R. (2019). Audiovisual media content preferences of children with autism spectrum disorders: Insights from parental interviews. Journal of Autism and Developmental Disorders. https://doi.org/10.1007/s10803-019-03987-1

Mazurek, M. O., \& Wenstrup, C. (2013). Television, video game and social media use among children with ASD and typically developing siblings. Journal of Autism and Developmental Disorders, 43(6), 1258-1271. https://doi.org/10.1007/s10803-012-1659-9

McMahon, C. M., \& Solomon, M. (2015). Brief report: Parent-adolescent informant discrepancies of social skill importance and social skill engagement for higher-functioning adolescents with autism spectrum disorder. Journal of Autism and Developmental Disorders, 45(10), 3396-3403. https://doi.org/10.1007/ s10803-015-2494-6

Metzger, M. J., Flanagin, A. J., Markov, A., Grossman, R., \& Bulger, M. (2015). Believing the unbelievable: Understanding young people's information literacy beliefs and practices in the United States. Journal of Children and Media, 9(3), 325-348.

Montes, G. (2016). Children with autism spectrum disorder and screen time: Results from a large, nationally representative US study. Academic Pediatrics, 16(2), 122-128. https://doi.org/10.1016/j. acap.2015.08.007

Odom, S. L., Thompson, J. L., Hedges, S., Boyd, B. A., Dykstra, J. R., Duda, M. A., Szidon, K. L., Smith, L. E., \& Bord, A. (2015). Technology-aided interventions and instruction for adolescents with autism spectrum disorder. Journal of Autism and Developmental Disorders, 45(12), 3805-3819.

Orsmond, G. I., \& Kuo, H. Y. (2011). The daily lives of adolescents with an autism spectrum disorder: Discretionary time use and activity partners. Autism, 15(5), 579-599. https://doi.org/10.1177/ 1362361310386503

Pardo, A., \& Siemens, G. (2014). Ethical and privacy principles for learning analytics. British Journal of Educational Technology, 45(3), 438-450. https://doi.org/10.1111/bjet.12152

Peterson, C. C., Garnett, M., Kelly, A., \& Attwood, T. (2009). Everyday social and conversation applications of theory-of-mind understanding by children with autism-spectrum disorders or typical development. European Child \& Adolescent Psychiatry, 18(2), 105-115. https://doi.org/10.1007/s00787-008-0711-y

Putnam, C., Hanschke, C., Todd, J., Gemmell, J., \& Kollia, M. (2019). Interactive technologies designed for children with autism: Reports of use and desires from parents, teachers, and therapists. ACM Transactions on Accessible Computing (TACCESS), 12(3), $1-37$.

Radesky, J. S., Schumacher, J., \& Zuckerman, B. (2015). Mobile and interactive media use by young children: The good, the bad, and the unknown. Pediatrics, 135(1), 1-3. https://doi.org/10.1542/ peds.2014-2251

Rankin, J. A., Weber, R. J., Kang, E., \& Lerner, M. D. (2016). Parentand self-reported social skills importance in autism spectrum disorder. Journal of Autism and Developmental Disorders, 46(1), 273-286. https://doi.org/10.1007/s10803-015-2574-7

Rao, P. A., Beidel, D. C., \& Murray, M. J. (2008). Social skills interventions for children with Asperger's syndrome or high-functioning autism: A review and recommendations. Journal of Autism 
and Developmental Disorders, 38(2), 353-361. https://doi.org/ 10.1007/s10803-007-0402-4

Reichow, B., \& Volkmar, F. R. (2010). Social skills interventions for individuals with autism: Evaluation for evidence-based practices within a best evidence synthesis framework. Journal of Autism and Developmental Disorders, 40(2), 149-166. https://doi.org/ 10.1007/s10803-009-0842-0

Rideout, V. J. (2019). The common sense census: Media use by tweens and teens. Common Sense Media Incorporated.

Ringland, K. E., Boyd, L., Faucett, H., Cullen, A. L. L., \& Hayes, G. R. (2017). Making in minecraft. Proceedings of the 2017 Conference on Interaction Design and Children-IDC '17. https://doi.org/10. 1145/3078072.3079749

Sanders, E. B. N. (2002). From user-centered to participatory design approaches. Design and the social sciences (pp. 18-25). CRC Press.

Schlosser, R. W., Brock, K. L., Koul, R., Shane, H., \& Flynn, S. (2019). Does animation facilitate understanding of graphic symbols representing verbs in children with autism spectrum disorder? Journal of Speech, Language, and Hearing Research, 62(4), 965-978. https://doi.org/10.1044/2018_jslhr-1-18-0243

Shane, H. C., \& Albert, P. D. (2008). Electronic screen media for persons with autism spectrum disorders: Results of a survey. Journal of Autism and Developmental Disorders, 38(8), 1499-1508. https://doi.org/10.1007/s10803-007-0527-5

Spiel, K., Frauenberger, C., Keyes, O., \& Fitzpatrick, G. (2019). Agency of autistic children in technology research-a critical literature review. ACM Transactions on Computer-Human Interaction (TOCHI), 26(6), 1-40. https://doi.org/10.1145/3344919

Stiller, A., \& Mößle, T. (2018). Media use among children and adolescents with autism spectrum disorder: A systematic review. Review Journal of Autism and Developmental Disorders, 5(3), 227-246. https://doi.org/10.1007/s40489-018-0135-7

Stone, B. G., Mills, K. A., \& Saggers, B. (2019). Online multiplayer games for the social interactions of children with autism spectrum disorder: A resource for inclusive education. International Journal of Inclusive Education, 23(2), 209-228. https://doi.org/ $10.1080 / 13603116.2018 .1426051$
Valkenburg, P. M., \& Cantor, J. (2000). Children's likes and dislikes of entertainment programs. In D. Zillmann \& P. Vorderer (Eds.), Media entertainment: The psychology of its appeal (pp. 135-152). Erlbaum.

van Schalkwyk, G. I., \& Dewinter, J. (2020). Qualitative research in the Journal of Autism and Developmental Disorders. Journal of Autism and Developmental Disorders, 50, 1-3.

Vygotsky, L. S. (1978). Mind in society: The development of higher mental processes. Harvard University Press. Original work published in 1933.

Whyte, E. M., Smyth, J. M., \& Scherf, K. S. (2015). Designing serious game interventions for individuals with autism. Journal of Autism and Developmental Disorders, 45(12), 3820-3831. https://doi.org/ 10.1007/s10803-014-2333-1

Wilson, B. J. (2008). Media and children's aggression, fear, and altruism. The Future of Children, 18(1), 87-118. https://doi.org/10. 1353 /foc. 0.0005

World Health Organization. (2020). Adolescent health. Health Topics. Accessed online on January 10, 2021. https://www.who.int/healthtopics/adolescent-health

Zhu, R., Hardy, D., \& Myers, T. (2019, December). Co-designing with adolescents with autism spectrum disorder: From ideation to implementation. In Proceedings of the 31st Australian Conference on Human-Computer-Interaction (pp. 106-116). https://doi. org/10.1145/3369457.3370914

Zi, X., Li, S., Rashedi, R., Rushdy, M., Lane, B., Mishra, S., et al. (2020). Adapting educational technologies across learner populations: A usability study with adolescents on the autism spectrum. In S. Deniso, M. Mack, Y. Xu \& B. C. Armstrong (Eds.), Proceedings of the 42nd Annual Conference of the Cognitive Science Society (pp. 1922-1928). Cognitive Science Society.

Publisher's Note Springer Nature remains neutral with regard to jurisdictional claims in published maps and institutional affiliations. 